\title{
Adult attention-deficit/hyperactivity disorder is associated with reduced norepinephrine transporter availability in right attention networks: a $(\mathrm{S}, \mathrm{S})-\mathrm{O}-\left[{ }^{11} \mathrm{C}\right]$ methylreboxetine positron emission tomography study
}

\author{
Christine Ulke $\mathbb{D}^{1}$, Michael Rullmann $\mathbb{D}^{2}$, Jue Huang ${ }^{1}$, Julia Luthardt ${ }^{2}$, Georg-Alexander Becker², Marianne Patt ${ }^{2}$, \\ Philipp M. Meyer ${ }^{2}$, Solveig Tiepolt ${ }^{2}$, Swen Hesse ${ }^{2}$, Osama Sabri ${ }^{2}$ and Maria Strauß ${ }^{1}$
}

\begin{abstract}
The norepinephrine transporter (NET) has been suggested to play a critical role in attention-deficit/hyperactivity disorder (ADHD). In this prospective controlled study we tested the a-priori-hypothesis that central NET availability is altered in adult ADHD patients compared to healthy controls. Study participants underwent single positron emission tomography-magnetic resonance imaging (PET-MRI). MRI sequences included high resolution T1-MPRAGE data for regions of interest (ROI) delineation and voxel-based morphometry (VBM) and T2-weighted fluid-attenuated inversionrecovery for detection and exclusion of pathological abnormalities. NET availability was assessed by NET-selective (S,S)$\mathrm{O}-\left[{ }^{11} \mathrm{C}\right]$ methylreboxetine; regional distribution volume ratios (DVR) were calculated based on individual PET-MRI data co-registration and a multi-linear reference tissue model with two constraints (MRTM2; reference region: occipital cortex). VBM analysis revealed no difference in local distribution of gray matter between the 20 ADHD patients (9 females, age $31.8 \pm 7.9$ years, $488 \pm 8 \mathrm{MBq}$ injected activity) and the 20 age-matched and sex-matched control participants ( 9 females, age $32.3 \pm 7.9$ years, $472 \pm 72 \mathrm{MBq}$ ). In mixed-model repeated-measures analysis with NET availability as dependent and $\mathrm{ROI}$ as repeated measure we found a significant main effect group in fronto-parietalthalamic-cerebellar regions (regions on the right: $F_{1,25}=12.30, p=.002$; regions on the left: $F_{1,41}=6.80, p=.013$ ) indicating a reduced NET availability in ADHD patients. None of the other investigated brain regions yielded significant differences in NET availability between groups after applying a Benjamini-Hochberg correction at a significance level of 0.05. Overall our findings demonstrate the pathophysiological involvement of NET availability in adult ADHD.
\end{abstract}

\section{Introduction}

Adult attention-deficit/hyperactivity disorder (ADHD) is an underrecognized chronic disorder with childhood-

\footnotetext{
Correspondence: Christine Ulke (Christine.Ulke@medizin.uni-leipzig.de)

${ }^{1}$ Department of Psychiatry and Psychotherapy, University of Leipzig Medical Center, 04103 Leipzig, Germany

${ }^{2}$ Department of Nuclear Medicine, University of Leipzig Medical Center, 04103 Leipzig, Germany

These authors contributed equally: Christine Ulke, Michael Rullmann
}

onset characterized by attention deficit, impulsivity and hyperactivity. The worldwide prevalence of ADHD is estimated to be $2.8 \%{ }^{1}$, in Germany as high as $4.7 \%^{2}$. Moreover, a mortality rate of $5.8 \%$ has been found in ADHD, highest in individuals diagnosed in adulthood and mainly driven by deaths from unnatural causes, e.g., accidents $^{3}$. ADHD is often accompanied by other comorbidities, such as depression, anxiety and conduct disorder ${ }^{4}$. ADHD symptoms have a detrimental impact on

\section{(c) The Author(s) 2019}

(c) Open Access This article is licensed under a Creative Commons Attribution 4.0 International License, which permits use, sharing, adaptation, distribution and reproduction c. in any medium or format, as long as you give appropriate credit to the original author(s) and the source, provide a link to the Creative Commons license, and indicate if changes were made. The images or other third party material in this article are included in the article's Creative Commons license, unless indicated otherwise in a credit line to the material. If material is not included in the article's Creative Commons license and your intended use is not permitted by statutory regulation or exceeds the permitted use, you will need to obtain permission directly from the copyright holder. To view a copy of this license, visit http://creativecommons.org/licenses/by/4.0/. 
social, financial and professional functioning ${ }^{5}$-the risk of losing one's job for example is three times higher in ADHD. Not surprisingly, the economic impact of adult ADHD places a significant burden on society. The gold standard treatment is pharmacotherapy, but $30 \%$ of adult patients with ADHD do not respond to medication ${ }^{6,7}$.

Patients with ADHD show executive functioning deficits, impaired attention and behavioral control, relating to brain areas modulated by noradrenergic transmission ${ }^{8,9}$ and norepinephrine transporter (NET) expression. Animal models of ADHD demonstrate the involvement of the central locus-coeruleus norepinephrine (LC-NE) system in behavioral control and the attentive process ${ }^{10,11}$. Drugs modulating NE transmission and targeting NET, such as atomoxetine or methylphenidate, are effective in ADHD patients $^{6}$ and have been shown to significantly occupy NET in vivo at clinically relevant doses ${ }^{12,13}$. There is ample and clear evidence that the LC-NE system is involved in regulating wakefulness and arousal ${ }^{14,15}$ shown to be reduced in patients with adult $\mathrm{ADHD}^{16-18}$.

While NE is released throughout the entire mammalian brain, evidence from structural and functional neuroimaging studies suggests that there are right hemispheric deficits in ADHD, specifically reduced overall cerebral volume including fronto-parietal areas, basal nuclei, globus pallidus and cerebellum ${ }^{19}$. Moreover, studies show functional abnormalities in two distinct domaindissociated networks: (1) the fronto-parietal-thalamiccerebellar network for attention and (2) the fronto-basalganglia network for inhibition ${ }^{20-22}$. The normalizing effect of methylphenidate and atomoxetine on the right fronto-parietal-thalamic activation during sustained attention ${ }^{23}$, known to be challenging for patients with $\mathrm{ADHD}^{24}$, corroborates the validity of the first model, further supported by the benefits of stimulation of the right dorsolateral frontal cortex on symptoms of inattention in patients with adult $\mathrm{ADHD}^{25}$. Concerning the second model, evidence from lesion and imaging studies suggest a direct pathway from the right inferior frontal cortex to the basal ganglia ${ }^{26,27}$ in stopping tasks. Importantly, dysfunctions of the prefrontal-striatal systems are implied in $\mathrm{ADHD}^{28-31}$ and basal ganglia are areas of NET expression, albeit less dense than in the $\mathrm{LC}^{32,33}$.

So far, changes in NET availability have not been described in adults with ADHD. One study exploring NET binding potential using radioligand $(\mathrm{S}, \mathrm{S})-\left[{ }^{18} \mathrm{~F}\right]$ FMeNER- $\mathrm{D}_{2}{ }^{33}$ in selected regions of interest (ROI) did not yield any significant differences between adults ADHD patients and healthy controls. However, using NET-selective (S,S)-O- $\left[{ }^{11} \mathrm{C}\right]$ methylreboxetine, a previous study found associations between central NET availability and trait impulsivity in twenty healthy individuals ${ }^{34}$.

In the present study we tested the hypothesis that central NET availability is altered in adult patients with
ADHD compared to healthy control subjects. We further explored whether NET availability is associated with ADHD symptom severity, neuropsychological and neurophysiological measures. To evaluate differences in NET availability, PET and NET-selective (S,S)-O- $\left[{ }^{11} \mathrm{C}\right]$ methylreboxetine (MRB) was applied in unmedicated adult patients with ADHD and age-matched and sex-matched healthy controls. We applied a ROI approach, focusing on areas known to be functionally relevant for ADHD that are linked to the LC-NE system.

\section{Materials/subjects and methods Study participants}

The study was conducted in accordance with the ICH Guideline for Good Clinical Practice (GCP) and the declaration of Helsinki, and was approved by the local ethics committee (EC number 155/15-ff) and the German Bundesamt für Strahlenschutz/Federal Office for Radiation Protection (number Z 5-22461 2-2016-008). Between February and December 2018, outpatients (age $\geq 18$ ) meeting DSM-V criteria for ADHD based on mental status examination, semi-structured Diagnostic Interview for ADHD in adults (DIVA $2.0^{35}$, adult ADHD Self-Report Scale Symptom Checklist (ASRS $1.1^{36}$ ), German version of the Wender-Utah-Rating-Scale (WURS- $\mathrm{k}^{37} \geq 30$; or known ADHD diagnosis in childhood) were consecutively recruited from the Adult ADHD outpatient center of the Department of Psychiatry and Psychotherapy of Leipzig University. Ageand sex-matched healthy control participants were recruited from the local community through advertisements. Patients had to be free of any psychotropic medication (ADHD-specific medication, antidepressants, sedatives, z-hypnotics, neuroleptics) and drug use for at least one month prior to the first screening visit; controls had to be naïve to psychotropic medication and had to be free of any drug use for at least one month prior to the first screening visit. Healthy controls received financial reimbursement.

Written informed consent was obtained from all participants at the beginning of the first screening visit after ensuring that all participants were able to understand the study protocol. To evaluate the general health of all participants, physical and neurologic status and routine laboratory tests were performed. Patients with serious psychiatric (acute severe depressive episode, suicidality, psychotic symptoms, bipolar disorder, schizophrenia, psychosis), neurological (history of brain surgery, significant brain malformation or neoplasm, head injury, stroke, epilepsy, neurodegenerative disorder), and/or severe somatic comorbidities were excluded. Any contraindication to MRI (e.g., heart or brain pacemaker, metal implants in the head/neck) and pregnancy also lead to patients' exclusion. A multi-drug urine test was 
performed at the first screening. Women underwent a urine pregnancy test before the PET-MRI measurement.

\section{Image acquisition, image data processing, and quantification}

PET-MRI data were acquired on a hybrid PET-MRI device (Biograph mMR, Siemens, Erlangen) and processed as previously described ${ }^{38}$. MRI sequences included high resolution T1-MPRAGE data for ROI delineation, and voxel-based morphometry (VBM) and T2-weighted fluidattenuated inversion-recovery (FLAIR) for detection and exclusion of pathological abnormalities. Dynamic PET imaging was performed after intravenous bolus injection $(90 \mathrm{~s})$ of $480 \pm 51 \mathrm{MBq}\left[{ }^{11} \mathrm{C}\right] \mathrm{MRB}$ for $90 \mathrm{~min}$ (23 frames: $4 \times 15 \mathrm{~s}, \quad 4 \times 60 \mathrm{~s}, \quad 5 \times 120 \mathrm{~s}, 5 \times 300 \mathrm{~s}, 5 \times 600 \mathrm{~s})$. For attenuation correction we applied the MR-based $\mu$-map estimation by Catana et al. ${ }^{39}$. Using PMOD (version 3.5, PMOD Technologies, Zurich, Switzerland), PET data motion correction was performed, as well as the generation of the regional DVR maps. Accordingly, we applied the multi-linear reference tissue model with 2 constraints $\left(\mathrm{MRTM} 2^{40}\right)$ using a fixed clearance rate of $\mathrm{k}^{\prime}=$ $0.0238 \mathrm{~min}^{-1}$ and the occipital cortex serving as the reference region. We used PMOD's Neuro module for automatic individual ROI delineation of the merged Automated Anatomical Labeling $\left(\mathrm{AAL}^{41}\right)$ atlas. For the Harvard Ascending Arousal Network $\left(\mathrm{AAN}^{42}\right)$ probability atlas we calculated the inverse transformation matrix of the spatial normalization to the MNI-space based on the T1-MPRAGE in SPM12 (Wellcome Centre for Human Neuroimaging University College London https://www.fil. ion.ucl.ac.uk/spm/software/spm12/) and applied the inverse matrix to the AAN atlas to transfer the atlas to the participant's individual space.

For VBM analysis we used the CAT12 (http://www. neuro.uni-jena.de/cat/) running in SPM12. In short, individual T1 data were spatially normalized, segmented in gray matter, white matter and cerebrospinal fluid and smoothed using a Gaussian filter with $8 \mathrm{~mm}$ full-width half-maximum filter size.

\section{Questionnaires}

The DSM-IV subscales of the Conners' Adult ADHD Rating Scale Self-Report (CAARS-SR-long version ${ }^{43,44}$ ) were applied at the screening visit to assess ADHD symptom severity. The Structured Clinical Interview for DSM-IV Axis I and Axis II disorders ${ }^{45,46}$ was performed to exclude comorbid psychiatric disorders. IQ was determined with vocabulary-based IQ screening (Wortschatztest, $\mathrm{WST}^{47}$ ). Substance use was evaluated with Alcohol Use Disorders Identification Test ${ }^{48}$ and Drug Use Disorders Identification Test ${ }^{49}$. The Beck Depression Inventory-II $^{50}$ (BDI) was applied to assess depressive symptom severity.

\section{Neuropsychological measures}

The sustained attention subtest of the computerized test of attentional performance $\left(\mathrm{TAP}^{51}\right.$ ) was used to assess attention and behavioral control at the screening visit. In this 15 -min test $(3 \times 5$-min blocks), a sequence of stimuli ranging in color, shape, size and filling, is presented on the monitor. A target stimulus occurs whenever it corresponds in shape with the preceding stimulus (e.g., the same shape but with different color, size and filling). Participants were instructed to press a button as quickly as possible when the target stimulus appeared. The probability for the appearance of the target-stimuli was 12 percent (450 trials, 54 targets, stimulus onset asynchrony: $2 \mathrm{~s})$. Errors of omission were defined as number of targets that were presented but not responded to and were used as measure of sustained attention. Errors of commissions were defined as number of non-targets that were responded to and used as measure of behavioral control (inhibition/impulsivity).

\section{Neurophysiological EEG measures}

We utilized standardized low-resolution brain electromagnetic tomography (sLORETA) to estimate the prefrontal current density of the theta band $(6.5-8.0 \mathrm{~Hz})$. To calculate the current source density of prefrontal EEG theta we recorded EEG signals at the screening visit with $\mathrm{Ag} / \mathrm{AgCl}$ electrodes using a QuickAmp amplifier (Brain Product GmbH, Gilching, Germany) from 31 electrode positions according to 10-20 system under a 15-min resting condition. Impedance of each electrode was kept below $10 \mathrm{k} \Omega$. The raw EEG was firstly down sampled to $256 \mathrm{~Hz}$ and then a band pass filtering between 0.1 and $100 \mathrm{~Hz}$ was performed. For all participants, the first 5 minutes of entire EEG was segmented and imported into SLORETA, to analyze the cortical three-dimensional distribution of scalp current density ${ }^{52}$. Each EEG was processed via a sLORETA cross-spectrum analysis; the software then calculated, based on the cross-spectrum data for the theta band $(6.5-8.0 \mathrm{~Hz})$, the current source values for each voxel. The current density values in the right and left dorsolateral prefrontal cortices (indexed by Brodmann Area 9; BA9) were averaged over all voxels within this ROI.

\section{Statistical analysis}

To investigate whether there are significant differences between ADHD patients and healthy controls regarding demographic variables and clinical variables Chi-square tests, t-tests for independent sample comparison, or Mann-Whitney $U$ tests (dependent on scale of measurement and normality of data) were performed to identify relevant covariates. To determine whether the NET availability is different between ADHD patients and healthy controls mixed-model repeated-measures 
(MMRM) analyses were applied with NET availability as outcome and ROI as repeated factor, and an unstructured covariance matrix. Models were implemented with restricted maximum likelihood algorithm. Fixed class effects of group (patients versus controls), ROIs, the interaction of group and ROI, BDI score as covariate, as well as random effects for subjects, matched participants and intercept were included. Separate models for attention-(including superior frontal gyrus, precuneus, angular and supramarginal gyri, cerebellum with crus and thalamus) and behavioral control-related (including inferior frontal gyrus, anterior cingulate, supplementary motor area, nucleus caudate, putamen, pallidum) ROIs based on the AAL probability atlas were calculated for the right and left side, as well as a model for LC and Raphe Nuclei based on the AAN probability atlas. The statistical significance of the main effect group or the interaction group $\mathrm{x}$ ROI was utilized to determine whether the NET availabilities in selected ROIs are different across the groups. In cases of significant main effects or interactions, Spearman correlation analyses between the mean DVR of respective ROIs and clinical (T-scores of CAARS DSM-IV subscales inattention and hyperactivity/impulsivity), neuropsychological (T-scores of numbers of omission and commission errors during the 15 -min sustained attention test) and neurophysiological measures (EEG theta current source density of BA9) were conducted; in case of significant findings, group was included as covariate to estimate the effect of group. All statistical analyses were computed using SPSS, version 24.0 for Windows (IBM Corp., Armonk, NY, USA). The two-tailed significance level was set at $p=.05$. The corrected significance level for multiple tests according to Benjamini and Hochberg ${ }^{53}$ was $p=.014$.

We used VBM to investigate differences in local distribution of gray matter between ADHD patients and agematched and sex-matched healthy control participants. The modulated and spatially normalized gray matter images were fed into SPM12, where we applied a twosample $t$-test with total intracranial volume as a confound to correct for different brain sizes. All clusters with a combined threshold of $p<.001$ uncorrected at peak level and $p<.05$ family-wise error corrected at cluster level were considered as significantly different.

\section{Results \\ Description of sample}

The final study sample consisted of 20 adult patients with ADHD (11 males, age $31.8 \pm 7.9$ years, $488 \pm 8 \mathrm{MBq}$ injected activity) and 20 age- and sex-matched healthy controls (11 males, age $32.3 \pm 7.9$ years, $472 \pm 72 \mathrm{MBq}$ ). Sample characteristics are presented in Table 1. Groups did not differ in basic demographic and biological

Table 1 Demographic and clinical characteristics

\begin{tabular}{|c|c|c|c|c|}
\hline & ADHD $(n=20)$ & Control $(n=20)$ & All $(n=40)$ & $p$-value \\
\hline \multicolumn{5}{|l|}{ Characteristic } \\
\hline Number of males & $11(55 \%)$ & $11(55 \%)$ & $22(55 \%)$ & 1.000 \\
\hline Age (years) & $31.8(7.9)$ 22-49 & $32.3(7.9)$ 20-52 & $32.1(7.8) 20-52$ & .843 \\
\hline Weight (kg) & $80.4(14.0)$ 49-107.5 & 79.4 (11.16) 58-98 & $79.9(12.5)$ & .373 \\
\hline Height $(\mathrm{cm})$ & 174.8 (8.1) 156-187 & 175.3 (10.3) 156-196 & $175.1(0.9)$ & .213 \\
\hline Smoking status, yes/no & $7 / 13(35 / 65 \%)$ & 6/14 (30/70\%) & 13/27 (32.5/67.5\%) & .736 \\
\hline BMI $\left(\mathrm{kg} / \mathrm{m}^{2}\right)$ & $26.2(3.7) 19.5-32.7$ & 25.7 (2.0) 21.9-28.9 & $26.0(3.0)$ & .610 \\
\hline Handedness & & & & .198 \\
\hline Right handed & $17(85 \%)$ & $20(100 \%)$ & $37(92.5 \%)$ & \\
\hline Left handed & $2(10 \%)$ & $0(0 \%)$ & $2(5 \%)$ & \\
\hline Ambidextrous & $1(5 \%)$ & $0(0 \%)$ & $1(2.5 \%)$ & \\
\hline Beck depression inventory, sum score & $9.9(11.3) 0-40$ & $1.4(2.5) 0-9$ & $5.7(9.2)$ & $<.0001$ \\
\hline WST_IQ & $107.3(9.4) 95-133$ & 106.9 (8.1) 92-122 & $107.1(8.7)$ & .889 \\
\hline WURS-K, sum score & $42.3(9.5) 25-60$ & $9.6(8.5) 0-24$ & $25.9(18.8)$ & $<.0001$ \\
\hline CAARS DSM-IV Inattention, T-score & $84.4(6.7) 70-90$ & 44.5 (8.6) 36-66 & $64.4(21.6)$ & $<.0001$ \\
\hline CAARS DSM-IV hyperactivity/ impulsivity, T-score & 74.8 (12.1) 54-90 & $43.1(5.4) 35-54$ & $58.9(18.5)$ & $<.0001$ \\
\hline
\end{tabular}

Annotations: Entries are means ( \pm standard deviations) or numbers (\%) and range 
characteristics (all: $p>.10$ ), however, a significant between-group difference in BDI sum scores was found $(p<.0001)$. Thus, we decided to integrate BDI sum score in multivariate analysis.

\section{Description of distribution volume ratios DVRs}

Examples of parametric images of the DVRs in axial and sagittal view, together with their corresponding T1MPRAGE images, are presented in Fig. 1. In general, most of the investigated brain regions showed a tendency towards lower values in adult ADHD patients compared with healthy controls. NET-selective MRB density in ADHD and healthy controls was highest in the ascending arousal system (i.e., dorsal raphe $>$ median raphe $>\mathrm{LC}$ ) and the thalamus (please cf. Figs. 1 and 2).

\section{VBM analysis}

We found no difference in local distribution of gray matter between ADHD patients and control participants.

\section{NET availability ADHD patients vs. healthy controls Ascending arousal system}

MMRM analysis using AAN atlas-based ROIs of the ascending reticular activating system (left, right LC; dorsal, median raphe nuclei) indicated a significant main effect of ROI $\left(F_{3,38}=10.72 ; p<.001\right)$ and a significant interaction of group $\mathrm{x}$ ROI $\left(F_{3,38}=3.38 ; p=.028\right)$ which did not survive adjustment for multiple tests. No main effects were obtained for group $\left(F_{1,39}=0.91 ; p=.347\right)$ or BDI $\left(F_{1,37}=0.41 ; p=.525\right)$.

\section{Attention-related ROIs}

In the right fronto-parietal-thalamic-cerebellar ROIs, MMRM analysis revealed a main effect group $\left(F_{1,25}=\right.$ 12.30, $p=.002)$, a main effect of ROI $\left(F_{6,30}=143.48\right.$; $p<.001)$, but no main effect of BDI $\left(F_{1,21}=1.41 ; p=.245\right)$ or group $\times \mathrm{ROI}$ interaction $\left(F_{6,30}=1.74 ; p=.154\right)$. When analyzing an analogous model for the left side, a main effect of ROI $\left(F_{6,38}=118.77 ; p<.001\right)$ and a main effect group emerged $\left(F_{1,41}=6.80, p=.013\right)$, but no main effect of BDI $\left(F_{1,37}=1.29 ; p=.264\right)$ or group $\mathrm{x}$ ROI interaction $\left(F_{6,38}=1.46 ; p=.217\right)$.

\section{Behavioral control-related ROIs}

In the right fronto-basal-ganglia ROIs, MMRM analysis revealed a statistical trend for main effect group $\left(F_{1,41}=\right.$ $3.09, p=.086)$ and group $\times$ ROI interaction $\left(F_{5,38}=2.14\right.$; $p=.081)$, a main effect of ROI $\left(F_{5,38}=138.31 ; p<.001\right)$, but no main effect of BDI $\left(F_{1,37}=0.03 ; p=.868\right)$. The analogous model for the left side yielded a main effect of ROI $\left(F_{5,30}=104.18 ; p<.001\right)$ but no significant main

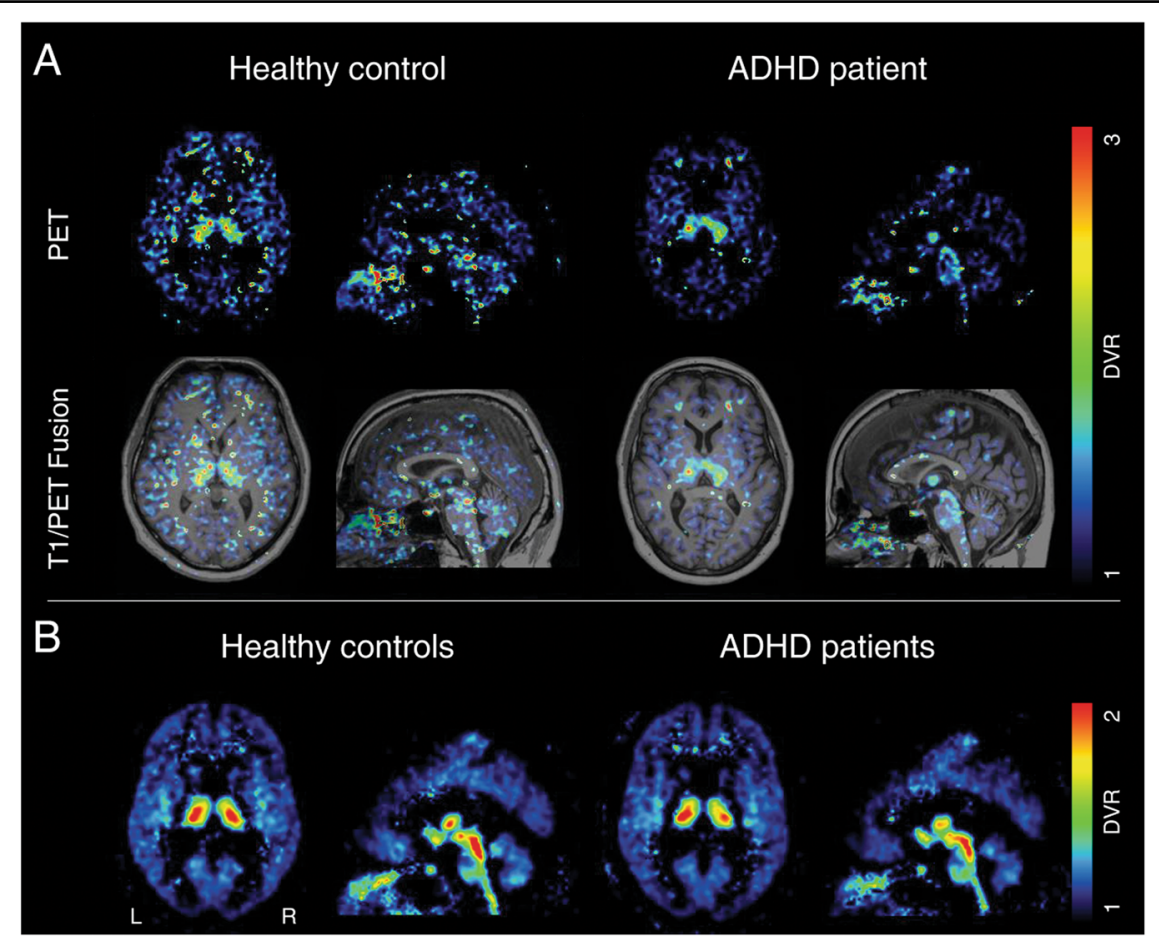

Fig. 1 Parametric images of the distribution volume ratio (DVR) in axial and sagittal view after spatial normalization to the MNI-space. a An exemplary healthy control and ADHD together with their corresponding T1-MPRAGE image. $\mathbf{b}$ Group mean of all healthy controls and ADHD patients. ADHD attention-deficit/hyperactivity disorder, MNI Montreal neurological institute, R right, L left. 


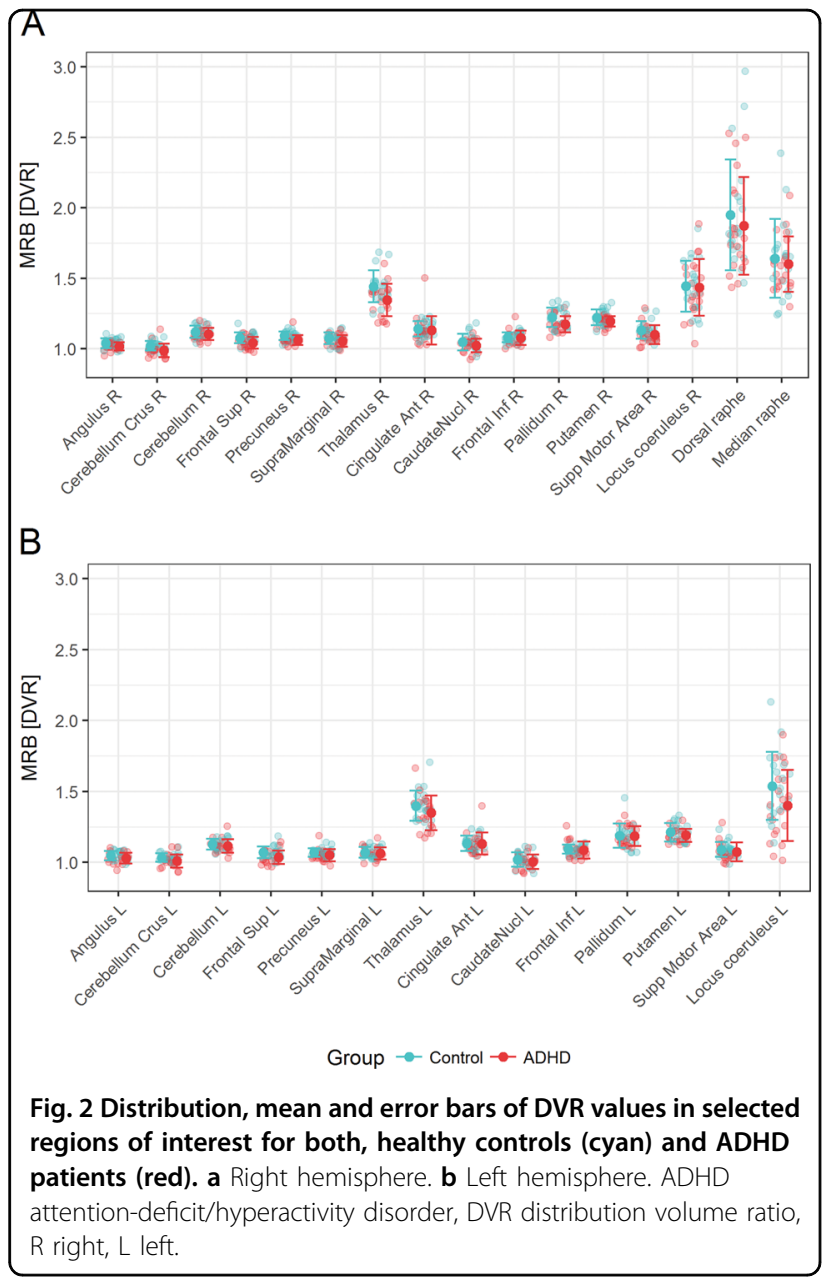

effect group, main effect BDI or group $\times$ ROI interaction $(.559 \leq p \leq .706)$.

\section{Exploratory correlation analysis \\ Mean DVR in attention-related ROls and ADHD symptom severity}

T-scores of CAARS DSM-IV subscales inattention and hyperactivity/impulsivity are presented in Table 1 . Correlation analysis revealed a significant negative association between mean DVR in the right fronto-parietalthalamic-cerebellar ROIs and ADHD symptom severity (inattention: rho $=-0.46 ; p=.003$; impulsivity/hyperactivity: rho $=-0.40 ; p=.011$ ); participants with lower NET availability scored higher on the CAARS DSM-IV scores, indicating higher symptom severity. The correlation coefficients decreased to -0.01 , and 0.10 , respectively, when group was included as covariate, which indicated a medium to large size effect of group. Analogous analysis for the left side did not yield any significant findings (inattention: rho $=-0.29 ; p=.068$; impulsivity/ hyperactivity: rho $=-0.34 ; p=.034$ ) after adjustment for multiple tests.

\section{Mean DVR in attention-related ROls and neuropsychological measures}

$T$-scores of omission and commission errors during the 15-min sustained attention test were calculated (please cf. Fig. 3). Correlation analysis revealed a significant association between mean DVR in right fronto-parietalthalamic-cerebellar ROIs and omission errors $($ rho $=$ 0.43; $p=.007$ ); participants with lower NET availability had worse performance $(=$ more omission errors $)$ in the sustained attention test.

The correlation coefficient decreased to 0.28 when group was included as covariate, indicating that the effect of group was small. No significant correlations were obtained for mean DVR and commission errors (rho = $0.06 ; p=.732$ ). Analogous analysis for the left side did not survive the correction for multiple tests (omission: rho $=$ $0.35 ; p=.027)$ and was not significant regarding commission errors ( $(\mathrm{hho}=0.02 ; p=.885)$.

\section{Mean DVR in attention-related ROls and neurophysiological measures}

The current source density values of resting EEG theta in the right and left dorsolateral prefrontal cortices (indexed by current source density of right/left BA9) were calculated. Correlation analysis revealed a significant negative association between mean DVR in right fronto-parietal-thalamic-cerebellar ROIs and theta current density (rho $=-0.41 ; p=.009$ ) indicating that participants with lower NET availability had greater theta current density in the right BA9 (please cf. Fig. 4c). The correlation coefficient decreased to -0.32 when group was included as covariate, indicating that the effect of group was small. Analogous analysis for the left side did not survive the correction for multiple tests ( $r h o=-0.31$; $p=.051)$.

\section{Discussion}

Our main goal was to test the hypothesis that central NET availability is altered in unmedicated adult patients with ADHD compared to age- and sex-matched healthy control subjects. We further explored whether NET availability is associated with ADHD symptom severity or neuropsychological and neurophysiological measures. We obtained several noteworthy findings. In line with our hypothesis, we demonstrated that patients with adult ADHD have decreased NET availability in relevant ROIs for attention, more pronounced in the right hemisphere, in comparison to healthy controls. In addition, in exploratory analyses we showed that lower NET availability in right fronto-parietal-thalamic-cerebellar ROIs is associated with more omission (but not commission) errors in a sustained attention test and greater EEG theta current density in the right dorsolateral prefrontal cortex. 

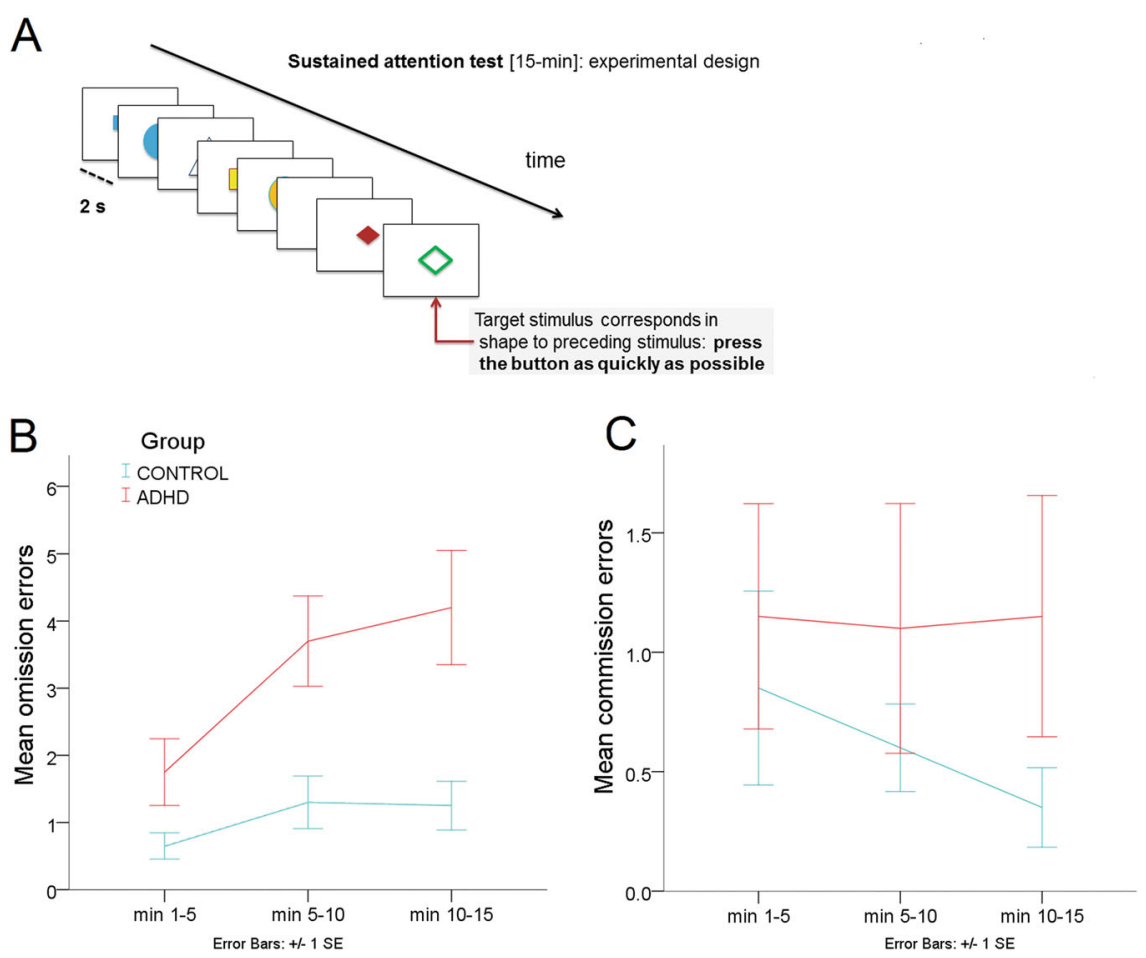

Fig. 3 The sustained attention subtest of the computerized test of attentional performance (TAP, Zimmermann and Fimm, 2012). a Experimental design. Stimulus onset asynchrony $=2 \mathrm{~s}$. $\mathbf{b}$ Mean omission errors during the 15 -min test in adult ADHD patients $(n=20)$, and ageand sex-matched healthy controls $(n=20)$. c Mean commission errors during the 15-min test in adult ADHD patients $(n=20)$ and healthy controls $(n=20)$.
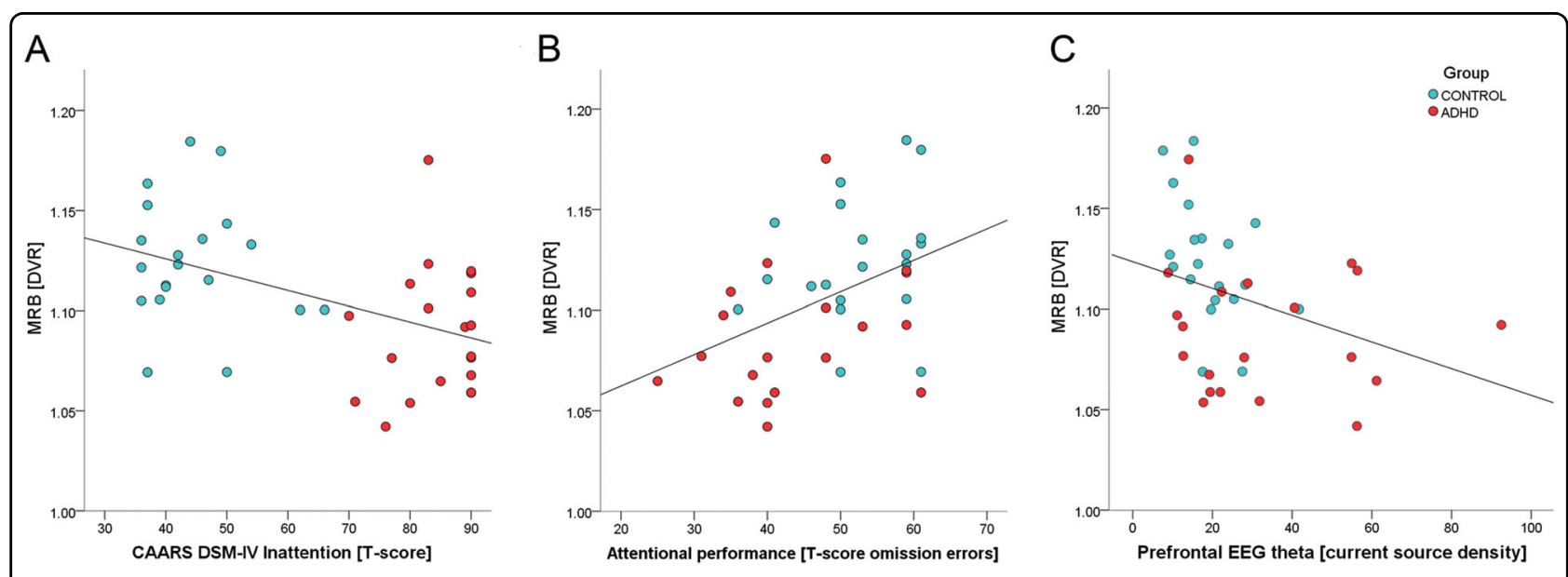

Fig. 4 Scatterplots of mean distributed volume ratios (DVR) in right fronto-parietal-thalamic-cerebellar regions of interest versus measures of inattention and EEG extracted theta current density. a Inattention assessed with the DSM-IV subscale inattention (total T-score) of the Conners' Adult ADHD Rating Scale Self-Report (CAARS-SR-long version). $\mathbf{b}$ Inattention assessed with the 15-min subtest of the computerized test of attentional performance (TAP; Zimmermann and Fimm, 2012). T-scores of omission errors (number of targets that were presented but not responded to) were used as a measure of sustained attention. c Mean absolute right BA9 theta $(6.5-8.0 \mathrm{~Hz})$ current density. Red circles: adult ADHD patients $(n=20)$; cyan circles: sex-matched and age-matched healthy controls $(n=20)$. BA Brodmann area.

Importantly, we found no differences in local gray matter distribution, which is in line with the systematic review by de Melo et al. ${ }^{19}$. The authors analyzed 17 reviews of whom none reported structural changes in adults with ADHD, while changes were reported in ADHD children. Thus, the obtained differences in NET 
availability between groups cannot be attributed to structural volume changes in our sample of adult ADHD patients, but rather suggest the involvement of the central LC-NE system corroborating the hypothesis of ADHD as a noradrenergic disorder. This is further supported by the fact that the LC-NE system mediates arousal ${ }^{11,15}$, which is impaired in adult ADHD, as shown in many EEGstudies of brain arousal ${ }^{16,17,54}$. Moreover, drugs, such as methylphenidate and atomoxetine, that modulate the LCNE system ${ }^{12}$, are effective in ADHD, as demonstrated in randomized controlled clinical trials ${ }^{55,56}$. It is of note that a recent placebo-controlled double-blind fMRI study, testing the comparative neurofunctional effects of methylphenidate and atomoxetine during sustained attention, showed normalization in right fronto-parietalthalamic areas under methylphenidate, but not under placebo $^{23}$. In line with these findings, in the current study the associations between NET availability in right frontoparietal-thalamic-cerebellar regions and neuropsychological and neurophysiological parameters, suggest a pathophysiological role of NET availability in adult ADHD and support the model of right hemisphere deficits $^{19,57}$ in ADHD. While brain arousal has been understood as a generalized state of the brain ${ }^{58}$, it is a consistent finding that the ability to maintain an alert state relies heavily on the right cerebral hemisphere $^{14,59,60}$. Our findings of greater right-frontal theta current density in individuals with lower NET availability in attention-related right-hemispheric regions point to a NET-related origin of this deficit. Interestingly, early lesion studies in rats revealed a right hemisphere bias in the LC-NE system ${ }^{61,62}$ indicating a top-down regulation of the NE activation by the right frontal cortex since lesions in this area resulted in lower NE levels in both hemispheres and the LC.

However, functional changes in fronto-basal-ganglia regions on the right side have equally been suggested in ADHD, more often in children ${ }^{31}$, which we could not confirm in the current study of adult ADHD patients. It is conceivable that the fronto-striatal deficits in ADHD children are due to the overall maturation delay ${ }^{31}$ and no longer present in adult ADHD patients. Our findings also do not replicate the findings of Vanicek et al. who found no significant differences in NET binding potential between ADHD and HC using (S,S)- $\left[{ }^{18} \mathrm{~F}\right]$ FMeNER-D2 between 22 ADHD patients und $22 \mathrm{HC}^{33}$. Similar to this study, we did not find any differences in NET availability in key regions of the ascending arousal system (i.e., right and left LC). One reason may be that the spatial resolution of the applied method reaches its limitation when quantifying small brain regions such as the LC. As seen in Fig. 2b, the parametric images of the DVRs are able to clearly delineate the hypothalami, while the LC is conflated with the raphe nuclei. In contrast to our approach, the study by Vanicek et al., besides using a different tracer, did not examine any cortical regions, known to be impaired in adult $\mathrm{ADHD}^{20}$ and their analyses did not consider the effect of hemisphere. Therefore, the question of consistency cannot be conclusively clarified given the differences in study design.

\section{Limitations}

Some limitations have to be mentioned. The relatively low spatial resolution of PET may have led to a DVR underestimation in the LC. The choice of specific templates (AAL, AAN) may have influenced the results, other parcellation schemes may generate different results, reducing the generalizability of our findings. However, the regional distribution of $\left[{ }^{11} \mathrm{C}\right] \mathrm{MRB}$ in the current study is consistent with the known distribution of NET in the brain and in line with previous studies reporting highest distribution in midbrain raphe, the thalamus and the $\mathrm{LC}^{63-65}$. Despite these limitations, our results warrant further studies to explore how NE/NET modulates resting-state activity (simultaneously measured by means of echo planar MR sequences) and appropriate behavior as assessed by thorough neuropsychological testing.

\section{Conclusion}

For the first time, the current study demonstrates decreased NET availability in adult ADHD patients in specific brain areas. Our findings indicate the pathophysiological involvement of NET availability in adult ADHD and warrant further clinical intervention studies targeting these specific areas.

\section{Acknowledgements \\ This study was funded by the Roland Ernst Foundation, Project 04/17.}

\section{Author's contributions}

Drs. C.U. and M.R. contributed equally to this work and served as co-first authors. Dr. C.U. had full access to all the data in the study and takes responsibility for the integrity of the data and the accuracy of the data analysis. Dr. M.R. had access to all the PET-MRI data and takes responsibility for the integrity and the accuracy of the modeling of the PET-MRI data. Study concept and design: M.S., S.H., O.S., M.R., C.U. Acquisition, analysis, or interpretation of data: All authors. Drafting of the paper: C.U., J.H. and M.R. Critical revision of the paper for important intellectual content: All authors. Statistical analysis: C.U. and M.R. Obtained funding: M.S. Administrative, technical, or material support: J.H., J.L., A.B., M.P., M.M., S.T. Supervision: M.S., S.H., O.S.

Conflict of interest

The authors declare that they have no conflict of interest.

\section{Publisher's note}

Springer Nature remains neutral with regard to jurisdictional claims in published maps and institutional affiliations.

Received: 23 June 2019 Revised: 12 September 2019 Accepted: 3 October 2019

Published online: 15 November 2019 


\section{References}

1. Fayyad, J. et al. The descriptive epidemiology of DSM-IV Adult ADHD in the World Health Organization World Mental Health Surveys. ADHD Atten. Deficit Hyperactivity Disord. 9, 47-65 (2017)

2. de Zwaan, M. et al. The estimated prevalence and correlates of adult ADHD in a German community sample. Eur. Arch. Psychiatry Clin. Neurosci. 262, 79-86 (2012).

3. Dalsgaard, S., Østergaard, S. D., Leckman, J. F., Mortensen, P. B. \& Pedersen, M. G. Mortality in children, adolescents, and adults with attention deficit hyperactivity disorder: a nationwide cohort study. Lancet $\mathbf{3 8 5}$ 2190-2196 (2015).

4. Kessler, R. C. et al. The prevalence and correlates of adult ADHD in the United States: results from the National Comorbidity Survey Replication. Am. J. Psychiatry 163, 716-723 (2006).

5. Harpin, V., Mazzone, L., Raynaud, J.P., Kahle, J. \& Hodgkins, P. Long-term outcomes of ADHD: a systematic review of self-esteem and social function. J. Atten. Disord. 20, 295-305 (2016).

6. Faraone, S. V., Biederman, J., Spencer, T. J. \& Aleardi, M. Comparing the efficacy of medications for ADHD using meta-analysis. Med. Gen. Med. 8, 4 (2006).

7. Clemow, D. B. \& Walker, D. J. The potential for misuse and abuse of medications in ADHD: a review. Postgrad. Med. 126, 64-81 (2014).

8. Benarroch, E. E. The locus ceruleus norepinephrine system Functional organization and potential clinical significance. Neurology 73, 1699-1704 (2009).

9. Mueller, A., Hong, D. S., Shepard, S. \& Moore, T. Linking ADHD to the neural circuitry of attention. Trends Cogn. Sci. 21, 474-488 (2017).

10. Viggiano, D., Ruocco, L., Arcieri, S. \& Sadile, A. Involvement of norepinephrine in the control of activity and attentive processes in animal models of attention deficit hyperactivity disorder. Neural Plasticity 11, 133-149 (2004).

11. Oken, B., Salinsky, M. \& Elsas, S. Vigilance, alertness, or sustained attention: physiological basis and measurement. Clin. Neurophysiol. 117, 1885-1901 (2006).

12. Hannestad, J. et al. Clinically relevant doses of methylphenidate significantly occupy norepinephrine transporters in humans in vivo. Biol. Psychiatry 68, 854-860 (2010).

13. Ding, Y.-S. et al. Clinical doses of atomoxetine significantly occupy both norepinephrine and serotonin transports: implications on treatment of depression and ADHD. Neurolmage 86, 164-171 (2014).

14. Posner, M. I. \& Petersen, S. E. The attention system of the human brain. Annu. Rev. Neurosci. 13, 25-42 (1990).

15. Berridge, C. W. Noradrenergic modulation of arousal. Brain Res. Rev. 58, 1-17 (2008).

16. Strauß, M. et al. Brain arousal regulation in adults with attention-deficit/ hyperactivity disorder (ADHD). Psychiatry Res. 261, 102-108 (2018).

17. Geissler, J., Romanos, M., Hegerl, U. \& Hensch, T. Hyperactivity and sensation seeking as autoregulatory attempts to stabilize brain arousal in ADHD and mania? Atten. Deficit Hyperactivity Disord. 6, 159-173 (2014).

18. Barry, R. J., Clarke, A. R. \& Johnstone, S. J. A review of electrophysiology in attention-deficit/hyperactivity disorder: I. Qualitative and quantitative electroencephalography. Clin. Neurophysiol. 114, 171-183 (2003).

19. Vieira de Melo, B. B., Trigueiro, M. J. \& Rodrigues, P. P. Systematic overview of neuroanatomical differences in ADHD: definitive evidence. Dev. Neuropsychol. 43, 52-68 (2018).

20. Hart, H., Radua, J., Nakao, T., Mataix-Cols, D. \& Rubia, K. Meta-analysis of functional magnetic resonance imaging studies of inhibition and attention in attention-deficit/hyperactivity disorder: exploring task-specific, stimulant medication, and age effects. JAMA Psychiatry 70, 185-198 (2013).

21. Kucyi, A., Hove, M. J., Biederman, J., Van Dijk, K. R. \& Valera, E. M. Disrupted functional connectivity of cerebellar default network areas in attention-deficit/ hyperactivity disorder. Hum. Brain Mapp. 36, 3373-3386 (2015).

22. Scolari, M., Seidl-Rathkopf, K. N. \& Kastner, S. Functions of the human frontoparietal attention network: Evidence from neuroimaging. Curr. Opin. Behav. Sci. 1, 32-39 (2015).

23. Kowalczyk, O. S. et al. Methylphenidate and atomoxetine normalise frontoparietal activation in ADHD during sustained attention. 7th World Congress on ADHD (Lisbon, 2019).

24. Shaw, T. H., Curby, T. W., Satterfield, K., Monfort, S. S. \& Ramirez, R. Transcranial Doppler sonography reveals sustained attention deficits in young adults diagnosed with ADHD. Exp. Brain Res. 237, 511-520 (2019).

25. Cachoeira, C. T. et al. Positive effects of transcranial direct current stimulation in adult patients with attention-deficit/hyperactivity disorder-A pilot randomized controlled study. Psychiatry Res. 247, 28-32 (2017).
26. Aron, A. R. et al. Converging evidence for a fronto-basal-ganglia network for inhibitory control of action and cognition. J. Neurosci. 27, 11860-11864 (2007)

27. Aron, A. R., Behrens, T. E., Smith, S., Frank, M. J. \& Poldrack, R. A. Triangulating a cognitive control network using diffusion-weighted magnetic resonance imaging (MRI) and functional MRI. J. Neurosci. 27, 3743-3752 (2007).

28. Greene, D. J. et al. Developmental changes in the organization of functional connections between the basal ganglia and cerebral cortex. J. Neurosci. 34, 5842-5854 (2014).

29. Castellanos, F. X. et al. Quantitative brain magnetic resonance imaging in attention-deficit hyperactivity disorder. Arch. Gen. Psychiatry 53, 607-616 (1996).

30. Dalley, J. W., Mar, A. C., Economidou, D. \& Robbins, T. W. Neurobehavioral mechanisms of impulsivity: fronto-striatal systems and functional neurochemistry. Pharmacol. Biochem. Behav. 90, 250-260 (2008).

31. Rubia, K. Alegría, A. A. \& Brinson, H. Brain abnormalities in attention-deficit hyperactivity disorder: a review. Rev. Neurol. 58(Suppl 1), S3-S16 (2014).

32. Schou, $M$. et al. Post-mortem human brain autoradiography of the norepinephrine transporter using (S, S)-[18F] FMeNER-D2. Eur. Neuropsychopharmacol. 15, 517-520 (2005).

33. Vanicek, T. et al. The norepinephrine transporter in attention-deficit/hyperactivity disorder investigated with positron emission tomography. JAMA Psychiatry 71, 1340-1349 (2014).

34. Hesse, S. et al. The association between in vivo central noradrenaline transporter availability and trait impulsivity. Psychiatry Res. 267, 9-14 (2017).

35. Kooij J. S. Adult ADHD: Diagnostic Assessment and Treatment. (Springer Science and Business Media, 2012).

36. Kessler, R. C. et al. The World Health Organization Adult ADHD Self-Report Scale (ASRS): a short screening scale for use in the general population. Psychological Med. 35, 245-256 (2005).

37. Retz-Junginger, P. et al. Wender Utah rating scale. The short-version for the assessment of the attention-deficit hyperactivity disorder in adults. Der Nervenarzt 73, 830-838 (2002).

38. Hesse, S. et al. Central noradrenaline transporter availability in highly obese, non-depressed individuals. Eur. J. Nucl. Med. Mol. Imaging 44, 1056-1064 (2017).

39. Catana, C. et al. Toward implementing an MRI-based PET attenuationcorrection method for neurologic studies on the MR-PET brain prototype. J. Nucl. Med. 51, 1431-1438 (2010).

40. Ichise, $M$. et al. Linearized reference tissue parametric imaging methods: application to [11C]DASB positron emission tomography studies of the serotonin transporter in human brain. J. Cereb. Blood Flow. Metab. 23, 1096-1112 (2003).

41. Tzourio-Mazoyer, N. et al. Automated anatomical labeling of activations in SPM using a macroscopic anatomical parcellation of the MNI MRI singlesubject brain. Neurolmage 15, 273-289 (2002).

42. Edlow, B. L. et al. Neuroanatomic connectivity of the human ascending arousal system critical to consciousness and its disorders. J. Neuropathol. Exp. Neurol. 71, 531-546 (2012)

43. Christiansen, $H$. Conners Skalen zu Aufmerksamkeit und Verhalten für Erwachsene: deutschsprachige Adaption der Conners' Adult ADHD Rating Scales (CAARS TM) von C. Keith Conners. Drew Erhardt und Elisabeth Sparrow; CAARS. (Huber, 2014).

44. Christiansen, $\mathrm{H}$. et al. German validation of the Conners Adult ADHD Rating Scales (CAARS) II: reliability, validity, diagnostic sensitivity and specificity. Eur. Psychiatry. 27, 321-328 (2012).

45. Wittchen, H.-U., Wunderlich, U., Gruschwitz, S. \& Zaudig, M. SKID I. Strukturiertes Klinisches Interview für DSM-IV. Achse I: Psychische Störungen. Interviewheft und Beurteilungsheft. Eine deutschsprachige, erweiterte Bearb. d. amerikanischen Originalversion des SKID I. (1997).

46. First, M. B., Gibbon, M., Spitzer, R. L., Williams, J. B. \& Benjamin, L. S. Structured clinical interview for DSM-IV axis II personality disorders (SCID-II). Am. Psych. Pub. (1997) (American Psychiatric Publishing, Inc., Arlington, VA, 1992). https:// openscholarship.wustl.edu/bsltests/2661/.

47. Schmidt, K. \& Metzler, P. Wortschatztest (WST). (Beltz, Weinheim, 1992).

48. Babor T. F., Higgins-Biddle J. C., Saunders J. B. \& Monteiro M. G. The alcohol use disorders identification test. In Guidelines for use in primary health care Geneva (World Health Organization, 1992).

49. Berman, A. H., Bergman, H., Palmstierna, T. \& Schlyter, F. Evaluation of the Drug Use Disorders Identification Test (DUDIT) in criminal justice and detoxification settings and in a Swedish population sample. Eur. Addiction Res. 11, 22-31 (2005). 
50. Beck, A. T., Ward, C. H., Mendelson, M. M., Mock, J. J. \& Erbaugh, J. J. An inventory for measuring depression. Arch. Gen. Psychiatry 4, 561-571 (1961).

51. Zimmermann, P. \& Fimm, B. Testbatterie zur Aufmerksamkeitsprüfung Version 2.3. Psytest: Herzogenrath (2013).

52. Pascual-Marqui, R. D., Esslen, M., Kochi, K. \& Lehmann, D. Functional imaging with low-resolution brain electromagnetic tomography (LORETA): a review. Methods Find. Exp. Clin. Pharmacol. 24(Suppl C), 91-95 (2002).

53. Benjamini, Y., Hochberg, Y. Controlling the false discovery rate: a practical and powerful approach to multiple testing. J. R. Stat. Soc. Ser. B 57, 289-300 (1995)

54. Hegerl, U. \& Hensch, T. The vigilance regulation model of affective disorders and ADHD. Neurosci. Biobehav. Rev. 44, 45-57 (2014).

55. Volkow, N. D. \& Swanson, J. M. Adult attention deficit-hyperactivity disorder New Engl. J. Med. 369, 1935-1944 (2013).

56. Faraone, S. V., Spencer, T., Aleardi, M., Pagano, C. \& Biederman, J. Meta-analysis of the efficacy of methylphenidate for treating adult attention-deficit/hyperactivity disorder. J. Clin. Psychopharmacol. 24, 24-29 (2004).

57. Sandson, T. A., Bachna, K. J. \& Morin, M. D. Right hemisphere dysfunction in ADHD: Visual hemispatial inattention and clinical subtype. J. Learn. Disabilities 33, 83-90 (2000).

58. Pfaff, D., Ribeiro, A., Matthews, J. \& Kow, L. M. Concepts and mechanisms of generalized central nervous system arousal. Ann. N.Y. Acad. Sci. 1129, 11-25 (2008).
59. Sturm, W. et al. Functional anatomy of intrinsic alertness: evidencefor a frontoparietal-thalamic-brainstem network in theright hemisphere. Neuropsychologia 37, 797-805 (1999).

60. Heilman, K. M., Valenstein, E. \& Watson, R. T. Neglect and related disorders. Proc. Semin. Neurol. 4, 209-219 (1984). (C) 1984 by Thieme Medical Publishers, Inc

61. Robinson, R. G. Lateralized behavioral and neurochemical consequences of unilateral brain injury in rats. In Cerebral Lateralization in Nonhuman Species. 135-156 (Elsevier, 1985)

62. Robinson, R. G. \& Coyle, J. T. The differential effect of right versus left hemispheric cerebral infarction on catecholamines and behavior in the rat. Brain Res. 188, 63-78 (1980)

63. Ding, $Y$. S. et al. PET imaging of the effects of age and cocaine on the norepinephrine transporter in the human brain using (S, S)-[11C] O-methylreboxetine and HRRT. Synapse 64, 30-38 (2010).

64. Chiang-shan, R. L. et al. Decreased norepinephrine transporter availability in obesity: positron Emission Tomography imaging with (S, S)-[11C] Omethylreboxetine. Neurolmage 86, 306-310 (2014).

65. Logan, J. et al. Imaging the norepinephrine transporter in humans with $(\mathrm{S}, \mathrm{S})$ [11C] O-methyl reboxetine and PET: problems and progress. Nucl. Med. Biol. 34, 667-679 (2007). 